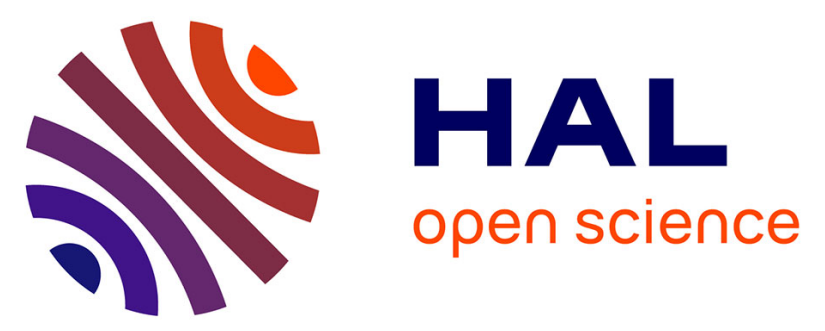

\title{
Temporal diffeomorphic Free Form Deformation (TDFFD) applied to motion and deformation quantification of tagged MRI sequences
}

Mathieu de Craene, C Tobon-Gomez, C Butakoff, Nicolas Duchateau, G Piella, K S Rhode, A Frangi

\section{To cite this version:}

Mathieu de Craene, C Tobon-Gomez, C Butakoff, Nicolas Duchateau, G Piella, et al.. Temporal diffeomorphic Free Form Deformation (TDFFD) applied to motion and deformation quantification of tagged MRI sequences. Statistical Atlases and Computational Models of the Heart (STACOM), MICCAI'11 Workshop, 2011, Toronto, Canada. pp.68-77, 10.1007/978-3-642-28326-0_7 . hal-02320745

\section{HAL Id: hal-02320745 \\ https://hal.science/hal-02320745}

Submitted on 19 Oct 2019

HAL is a multi-disciplinary open access archive for the deposit and dissemination of scientific research documents, whether they are published or not. The documents may come from teaching and research institutions in France or abroad, or from public or private research centers.
L'archive ouverte pluridisciplinaire $\mathbf{H A L}$, est destinée au dépôt et à la diffusion de documents scientifiques de niveau recherche, publiés ou non, émanant des établissements d'enseignement et de recherche français ou étrangers, des laboratoires publics ou privés. 


\title{
Temporal diffeomorphic Free Form Deformation (TDFFD) applied to motion and deformation quantification of tagged MRI sequences
}

\author{
M. De Craene ${ }^{a, b}$, C. Tobon-Gomez ${ }^{a, b}$, C. Butakoff ${ }^{a, b}$, N. Duchateau ${ }^{a, b}$, \\ G. Piella ${ }^{a, b}$, K.S. Rhode ${ }^{c}$, and A. F. Frangi ${ }^{a, b}$ \\ CISTIB, ${ }^{a}$ DTIC, Universitat Pompeu Fabra, and ${ }^{b}$ CIBER-BBN, Barcelona, Spain. \\ ${ }^{c}$ Division of Imaging Sciences and Biomedical Engineering, KCL, UK.
}

\begin{abstract}
This paper presents strain quantification results obtained from the Tagged Magnetic Resonance Imaging (TMRI) sequences acquired for the $1^{\text {st }}$ cardiac Motion Analysis Challenge (cMAC). We applied the Temporal Diffeomorphic Free Form Deformation (TDFFD) algorithm to the phantom and the 15 healthy volunteers of the cMAC database. The TDFFD was modified in two ways. First, we modified the similarity metric to incorporate frame to frame intensity differences. Second, on volunteer sequences, we performed the tracking backward in time since the first frames did not show the contrast between blood and myocardium, making these frames poor choices of reference.

On the phantom, we propagated a grid adjusted to tag lines to all frames for visually assessing the influence of the different algorithmic parameters. The weight between the two metric terms appeared to be a critical parameter for making a compromise between good tag tracking while preventing drifts and avoiding tag jumps. For each volunteer, a volumetric mesh was defined in the Steady-State Free Precession (SSFP) image, at the closest cardiac time from the last frame of the tagging sequence. Uniform strain patterns were observed over all myocardial segments, as physiologically expected.
\end{abstract}

\section{Introduction}

TMRI provides non invasively image markers for tracking tissue motion and deformation along the cardiac cycle. The introduction of CSPAMM [1] efficiently solved tag fading issues that were traditionally hampering the analysis of TMRI data in diastole. Breathing artifacts producing unaligned slices have been corrected by the introduction of navigator-driven protocols and the move to fully 3D acquisitions. In [3], a CSPAMM-based protocol was proposed by performing three acquisitions sequentially with line tag preparation in each orthogonal direction. Each of these three acquisitions is performed in a single breath-hold of 18 heartbeats duration and retrospectively corrected for misalignment using a respiratory navigator.

In this paper, we extend the TDFFD algorithm [4], initially designed for 3D US images quantification and applied it to the $1^{\text {st }}$ cMAC TMRI database. We 


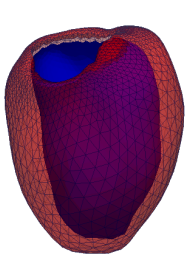

(a)

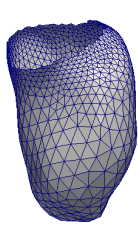

(b)

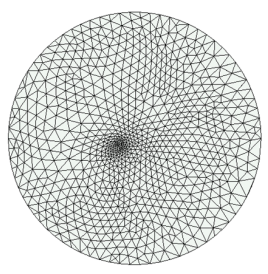

(c)

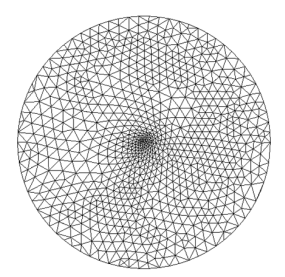

(d)

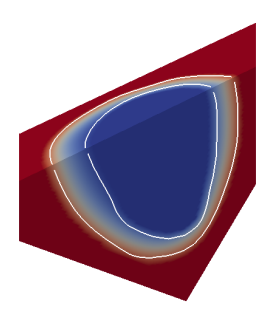

(i)

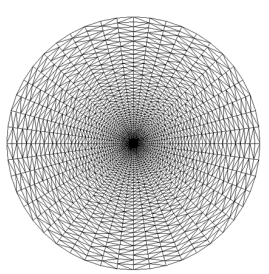

(e)

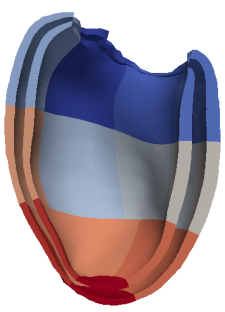

(j)

Fig. 1: Remeshing process: From (a) the LV mesh, extract (b) endocardial surface, and (c) map it to a disk, (d) correct the mapping by moving the apical point to the center, (e) create a new parametrization that maps the new vertices onto the surface and (f,g,h) add AHA regions, (i) solve Laplace equation in $3 \mathrm{D},(\mathrm{j})$ produce volumetric mesh with 3 layers of wedge elements (the middle layer was hidden for clarity)

present and discuss strain quantification results on the set of healthy volunteers and the phantom acquisitions.

\section{Methods}

\subsection{Preprocessing}

The cMAC database was acquired according to the imaging protocols defined in [5]. In this paper, we worked on the resampled grid TMRI images. For obtaining these fused images, the three images resulting from each acquisition direction were first resampled to a common reference space (usually taken as the short axis space) with isotropic voxel resolution. The geometric mean of intensities of the three resampled volumes were then computed at each voxel to produce the reconstructed TMRI images with a tagging grid pattern, as shown in Fig. 2(c,d).

\subsection{Segmentation}

Myocardial borders were defined on the reference image (here taken as the last frame of the sequence) by segmenting the SSFP images. The corresponding frame in the SSFP sequence was selected by looking for the closest trigger time. The left ventricular (LV) cavity was then segmented from the selected short axis dataset by a human observer experienced in cardiac magnetic resonance postprocessing. 


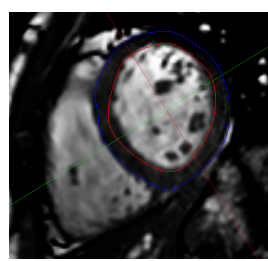

(a)

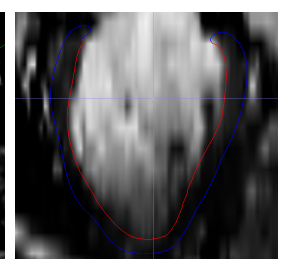

(b)

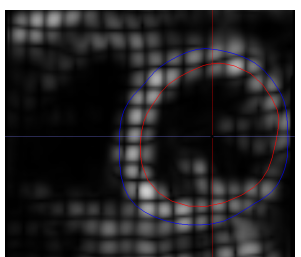

(c)

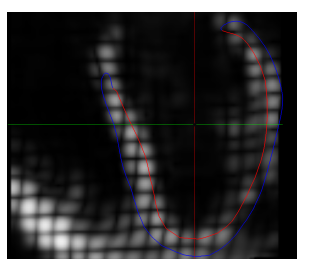

(d)

Fig. 2: Segmentation obtained from the SSFP short axis image (a,b) mapped using the DICOM transformation to the TMRI image $(\mathrm{c}, \mathrm{d})$

3D meshes of the LV were obtained by manually deforming a LV model [6]. Both the visualization and the segmentation was performed with GIMIAS v1.3.0 ${ }^{1}$. The manually segmented mesh was then registered to the coordinates of the TMRI sequence using DICOM header information. The result of mapping the SSFP segmentation on the TMRI image is shown in Fig. 2 for the first volunteer.

\subsection{Mesh postprocessing}

The goal of this postprocessing is to produce a mesh meeting the following requirements: 1) to provide a regular definition of the 17 American Heart Association (AHA) segments, 2) to provide a definition of the local radial direction that is consistent at endo- and epicardium and 3) to be a volumetric mesh for quantifying strain in the whole endocardium. We introduce here a two-step pipeline meeting these requirements.

Imposing a regular mesh structure for improved definition of AHA segments. Any surface, homeomorphic to a disk, can be mapped to a disk by requiring that every coordinate has a vanishing Laplacian. We want to compute a bijective mapping $\varphi: \mathcal{M} \rightarrow \mathcal{D} \subset \mathbb{R}^{2}$, where $\mathcal{M}$ is a surface and $\mathcal{D}$ is a disk. First we need to define the boundary $\partial \mathcal{D}$ of $\mathcal{D}$ by uniformly sampling a circle (a unit circle for simplicity), onto which we want to map the boundary $\partial \mathcal{M}$. Therefore, for every point of $\partial \mathcal{M}$ there is a corresponding point on the disk boundary $\partial \mathcal{D}$. The computed mapping will give us the coordinates of the remaining points inside the disk. Let us assume that the disk is in the XY coordinate plane, and the boundary coordinates are given by vector-columns $\mathbf{x}_{0}$ and $\mathbf{y}_{0}$ (concatenation of $x$ and $y$ coordinates of all boundary points). Let $\mathbf{L}_{\mathcal{M} \backslash \partial \mathcal{M}}$ be the Laplacian matrix of the mesh that represents the surface $\mathcal{M}$ with the rows corresponding to its boundary $\partial \mathcal{M}$ removed. Let $\mathbf{x}_{\partial \mathcal{D}}, \mathbf{y}_{\partial \mathcal{D}}, \mathbf{x}_{\mathcal{D} \backslash \partial \mathcal{D}}$ and $\mathbf{y}_{\mathcal{D} \backslash \partial \mathcal{D}}$ be the $x, y$ coordinates of the points on the disk (the ones which we are calculating and that define our mapping) corresponding to the boundary and the interior, respectively. Then, the following two systems of linear equations give the desired mapping (vectors $\mathbf{x}$ and $\mathbf{y})$, while the connectivity information is retained from the mesh representation

\footnotetext{
${ }^{1}$ http://www.gimias.org
} 

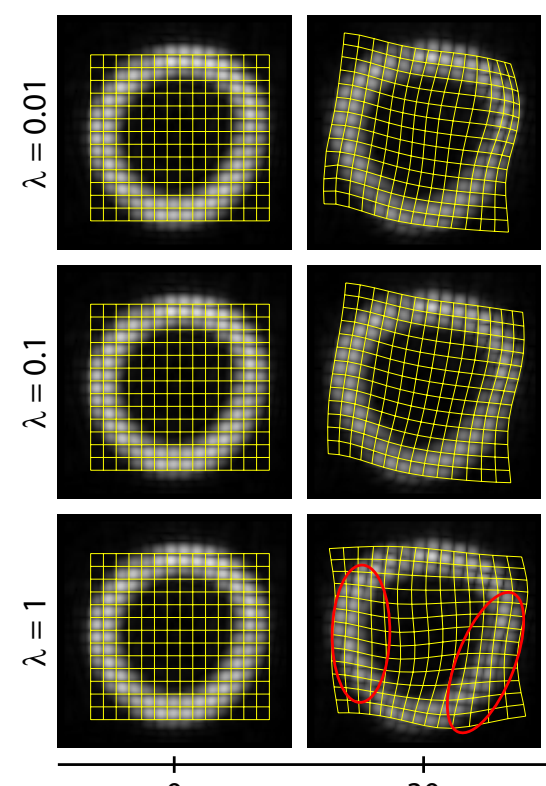

30
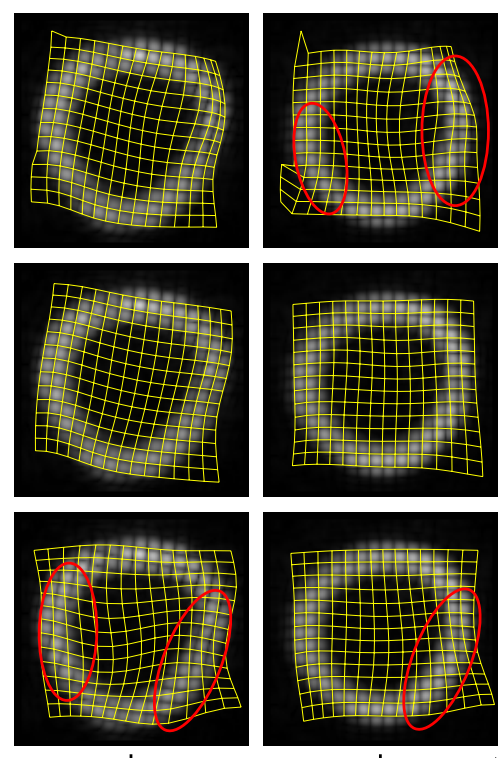

60

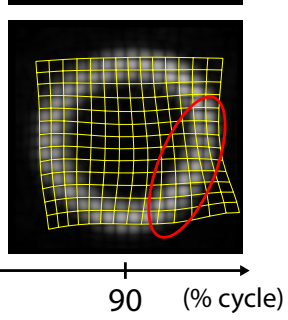

Fig. 3: Propagation of a synthetic grid using the TDFFD tracking results for 3 different $\lambda$ values in Eq. 2. Drift errors (top row) and tag jumps (bottom row) are highlighted using red ellipses.

of $\mathcal{M}[7]$ :

$$
\left\{\begin{array}{c}
\mathbf{L}_{\mathcal{M} \backslash \partial \mathcal{M}} \cdot \mathbf{x}_{\mathcal{D} \backslash \partial \mathcal{D}}=0 \\
\mathbf{x}_{\partial \mathcal{D}}=\mathbf{x}_{0}
\end{array} ; \quad\left\{\begin{array}{c}
\mathbf{L}_{\mathcal{M} \backslash \partial \mathcal{M}} \cdot \mathbf{y}_{\mathcal{D} \backslash \partial \mathcal{D}}=0 \\
\mathbf{y}_{\partial \mathcal{D}}=\mathbf{y}_{0}
\end{array}\right.\right.
$$

The above methodology provides a simple method for mapping endocardium to a disk, where the edge of the endocardium (Fig. 1b) is mapped to an uniformly sampled circumference of the disk (Fig. 1c). However, it is in our interest to map the cardiac apex to the center of the disk. We employ the mass spring model to displace the apical point to the center while uniformly spreading the cell deformation to all the cells (Fig. 1d). Given this mapping, we can impose a regular mesh structure by generating vertices as points of intersection of concentric circles and rays emanating from the center (Fig. 1e). It is easy now to compute a transformation between Fig. 1d and Fig. 1e through barycentric coordinates. This transformation allows us to generate a new mesh (Fig. 1g) whereupon it is easy to define the 17 AHA segments (Fig. 1f), therefore satisfying the first requirement.

Volumetric mesh generation by Laplace streamlines sampling. The generation of the volumetric mesh (third requirement) follows 4 steps:

- generate an image, between epi- and endocardium, that is a solution to Laplace equation $\Delta \Psi=0$, by successive over relaxation (see Fig. 1i, $\Delta$ is the Laplace operator) 


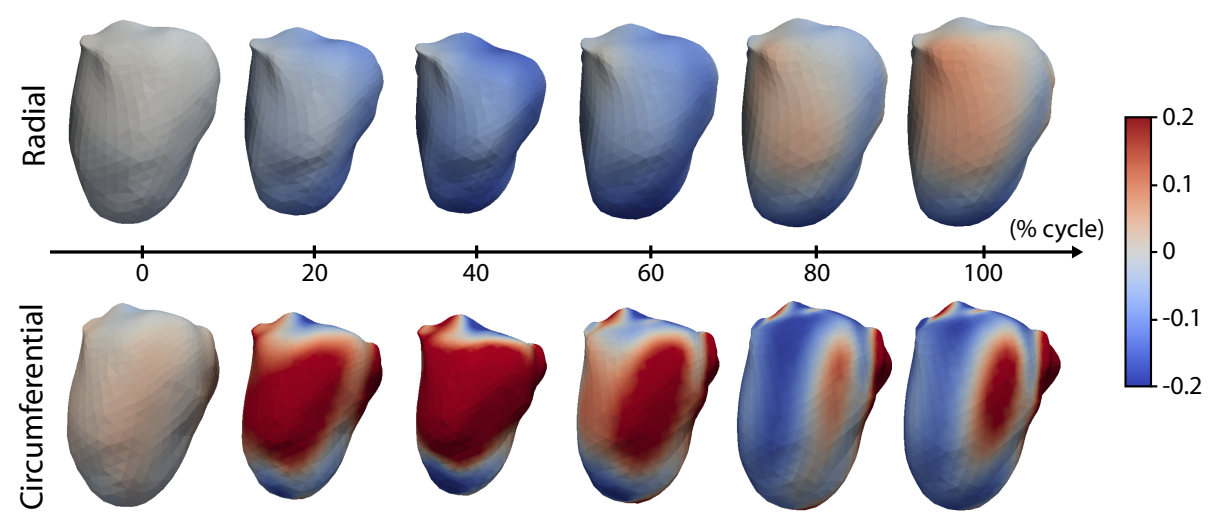

Fig. 4: Evolution of radial and circumferential strains for the first volunteer over the cardiac cycle, plotted using a colormap. An animated version of this figure is available at http://mathieu.decraene.info/stacom11/strain.gif.

- compute streamlines of the normalized gradient of $\Psi$ starting from each endocardial vertex towards the epicardial surface

- generate layers of vertices equidistantly along the line connecting the endpoints of the streamlines

- use the connectivity information of the endocardial surface mesh to generate 6-node wedge elements connecting all the layers (Fig. 1j)

At each node, the direction of the outgoing streamline is taken as radial direction. This ensures a consistent definition of the normal direction across the different 'layers' of the volumetric mesh (second requirement).

\subsection{Motion and Deformation Quantification}

The TDFFD algorithm described in [4] optimizes a 4D velocity field parametrized by B-Spline spatiotemporal kernels. The advantage of representing the velocity rather than the displacement is to introduce temporal consistency in the recovered transformation, i.e., that motion at a given time point depends on all previous times. In this paper, the TDFFD algorithm was modified in two aspects. First, the tracking was performed backward in time starting from the last image. This option was taken because in the first images of a TMRI sequence, the blood is magnetized in the same way as the tissue. The distinction between blood and myocardial borders is therefore invisible, making the first frames a poor choice of reference.

The second change affects the similarity metric. The original TDFFD similarity metric [4] sums the squared intensity differences between each image with respect to the first image in the sequence. This choice was shown to be efficient for limiting the accumulation of motion errors generating drift effects. However, quantifying similarity from frame to frame is expected to be more sensitive to small incremental displacements. It is also more robust to possible intensity 


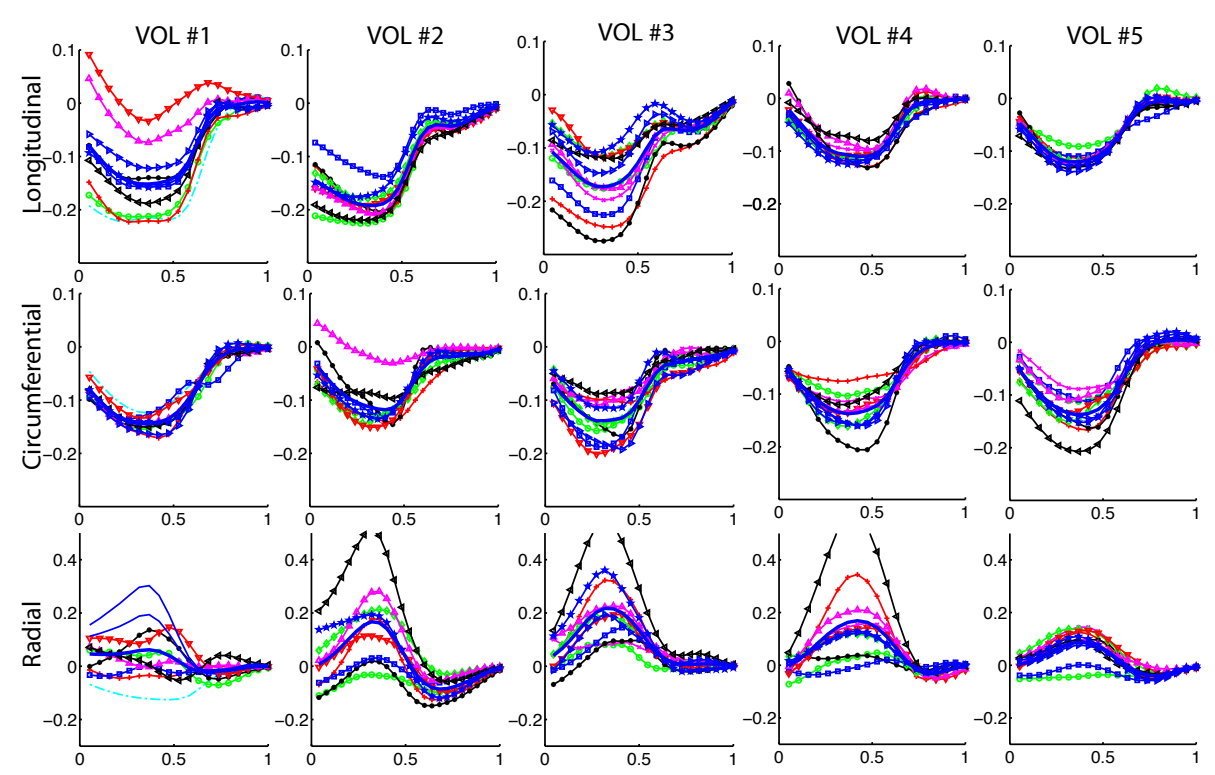

Fig. 5: Longitudinal, circumferential and radial strains for Volunteers \#1 to \#5 of the cMAC database plotted as a function of time (normalized by one heart period).

changes due to smooth alterations of tissue magnetization in time. For this reason, we extended the original metric by summing similarities quantified both from frame to frame and to the reference image. For this, we consider two sets of samples. The first set of samples is defined as $X=\left\{\mathbf{x}_{i}, i \in\left[1, N_{X}\right]\right\}$ where all $\mathbf{x}_{i}$ belongs to the space of coordinates of the reference image. The second set of samples is taken through the full $3 \mathrm{D}+\mathrm{t}$ space: $Y=\left\{\left(\mathbf{y}_{k}, f_{k}\right), k \in\left[1, N_{Y}\right]\right\}$ where each $\mathbf{y}_{k}$ belongs to the space of coordinates of a frame $f_{k} \in[2, F]$ that is part of the $Y$ samples set generation and $F$ is the number of frames in the sequence. The full metric is then defined as

$$
\begin{aligned}
M= & \sum_{i=1}^{N_{X}} \sum_{j=1}^{F-1}\left(I_{j}\left(\varphi_{F}^{j}\left(\mathbf{x}_{i} ; \mathbf{p}\right)\right)-I_{F}\left(\mathbf{x}_{i}\right)\right)^{2} \\
& +\lambda \sum_{k=1}^{N_{Y}}\left(I_{f_{k}-1}\left(\varphi_{f_{k}}^{f_{k}-1}\left(\mathbf{y}_{k} ; \mathbf{p}\right)\right)-I_{f_{k}}\left(\mathbf{y}_{k}\right)\right)^{2}
\end{aligned}
$$

where $\varphi_{m}^{n}(\mathbf{x})$ stands for the transport of a coordinate $\mathbf{x}$ at time $m$ to the time $n$ and $\lambda$ is a factor balancing the metric terms. This transport is made by following the flow of a continuous $3 \mathrm{D}+\mathrm{t}$ velocity field $\mathbf{v}(\mathbf{x}, t ; \mathbf{p})$, parametrized by B-Spline kernels, and controlled by the set of parameters $\mathbf{p}$ :

$$
\varphi_{m}^{n}(\mathbf{x} ; \mathbf{p})=\mathbf{x}+\int_{m}^{n} \mathbf{v}\left(\boldsymbol{\varphi}_{m}^{t}(\mathbf{x} ; \mathbf{p}), t ; \mathbf{p}\right) \mathrm{d} t
$$




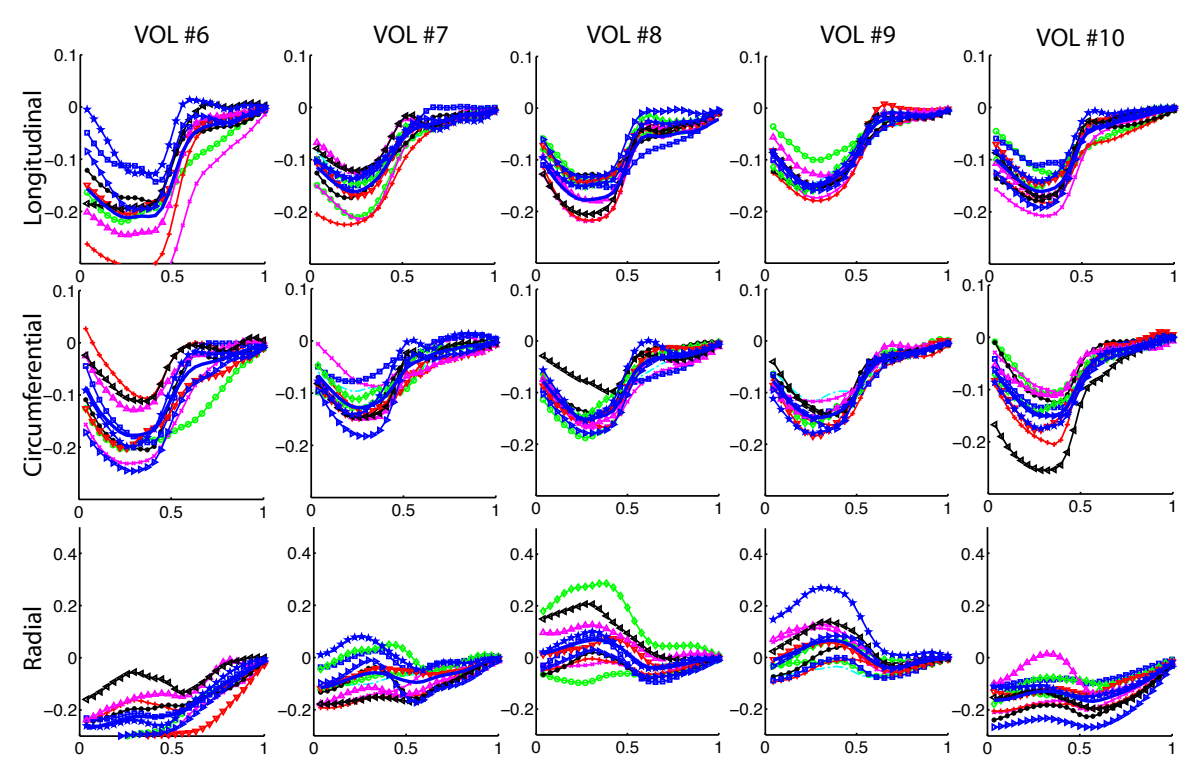

Fig. 6: Longitudinal, circumferential and radial strains for Volunteers \#6 to \#10 of the cMAC database plotted as a function of time (normalized by one heart period).

It was shown in [4] that for a time step $\Delta t<<1$, the derivative of $\varphi_{s}^{t+\Delta t}(\mathbf{x} ; \mathbf{p})$ w.r.t $\mathbf{p}$ can be computed from $\mathrm{d} \boldsymbol{\varphi}_{s}^{t}(\mathbf{x} ; \mathbf{p}) / \mathrm{d} \mathbf{p}$

$$
\frac{\mathrm{d} \boldsymbol{\varphi}_{s}^{t+\Delta t}(\mathbf{x} ; \mathbf{p})}{\mathrm{d} \mathbf{p}}=(\mathbf{I}+\mathbf{D} \mathbf{v}(\mathbf{x}, t ; \mathbf{p}) \Delta t) \frac{\mathrm{d} \boldsymbol{\varphi}_{s}^{t}(\mathbf{x} ; \mathbf{p})}{\mathrm{d} \mathbf{p}}+\frac{\partial \mathbf{v}(\mathbf{x}, t ; \mathbf{p})}{\partial \mathbf{p}} \Delta t
$$

By iterating Eq. 4 from $(s, t)=(m, m)$ to $(s, t)=(n-\Delta t, n)$, one can obtain the derivative of $\boldsymbol{\varphi}_{m}^{n}(\mathbf{x} ; \mathbf{p})$ and compute the total derivative of the metric given in Eq. 2 by application of the chain rule.

\subsection{Strain quantification}

Strain is computed in the space of coordinates of the last frame, being a good approximation of Lagrangian strain at end-diastole. The computation of the strain tensor involves the backward multiplication of spatial derivatives of the velocity fields, corresponding to the factor between brackets in the first term of Eq. 4 . The strain tensor is then projected on a set of local directions: radial, circumferential and longitudinal [4]. The radial direction is computed as described in Section 2.3. The longitudinal direction is taken as constant over the whole LV and is defined as the difference between the endocardial apex and the center of the mitral valve. The circumferential direction is computed as the cross product of the two other directions. 


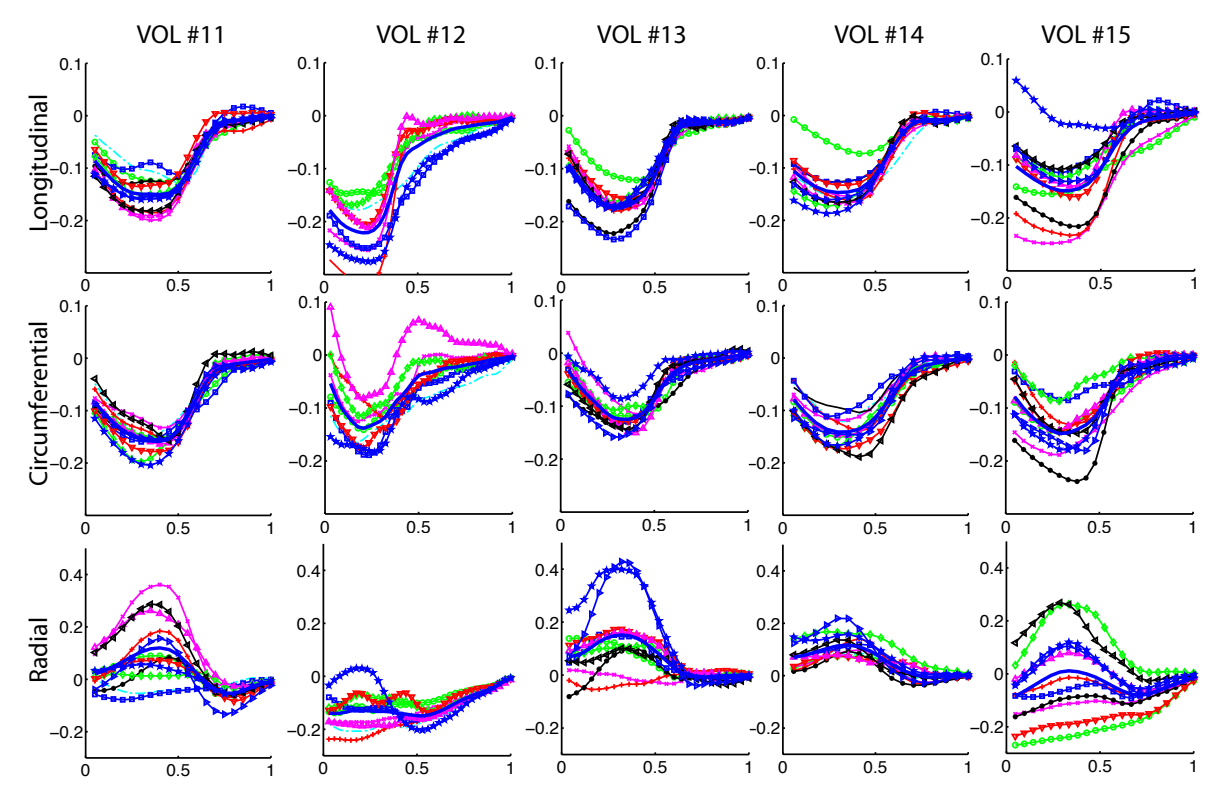

Fig. 7: Longitudinal, circumferential and radial strains for Volunteers \#11 to \#15 of the cMAC database plotted as a function of time (normalized by one heart period).

\section{Experiments}

In-vitro phantom. Fig. 3 shows the propagation of a regular grid from the first frame to each image of the sequence for one slice of the phantom sequence. The B-Spline velocity grid had a resolution of 4 control points in each dimension. The number of samples $N_{X}$ and $N_{Y}$ were chosen to represent about $15 \%$ of the total number of voxels. The $\lambda$ weight in Eq. 2 was adjusted by visual inspection of the tracking results. Fig. 3 shows that for low values of $\lambda$, i.e. when the frame to frame term of the metric predominates, TDFFD efficiently tracks tags crossing at mid-cycle but suffers from drift in the last frame (see red ellipses in first row, last column). Alternatively, when giving too much importance to the nonsequential term, the tracking suffers from tag jumps (e.g red ellipses in the last row). In this experiment, we found a value of $\lambda=0.1$ qualitatively optimal for weighting the two terms in Eq. 2.

Healthy volunteers. Motion and strain were quantified using the modified TDFFD algorithm (Section 2.4 and 2.5) and the set of local directions computed at each node of the volumetric mesh (Section 2.3). Each of the three components of strain was averaged across 12 AHA regions (corresponding to basal and midregions) and plotted for all patients of the cMAC database in Fig. 5, 6 and 7 . The recovered strain curves showed a similar pattern in all volunteers, in good agreement with clinical literature [2]. It is expected in healthy volunteers that all regions contract synchronously with a similar strain amplitude. Fig. 5, 6 and 7 show that strain curves were in general uniform over all segments for each 
patient, except for radial strain. Although radial strain was reported to show the highest variability [2], the dispersion of radial strain in Fig. 5 is more likely due to the smaller number of tags in the radial direction (between 2 and 3 tags). An alternative way of plotting strain is to show on a surface mesh and for a given cardiac time the value of one strain component using a color map. This representation mode is used in Fig. 4 for the first volunteer. Drift errors in the radial strain values are visible at the end of the cycle.

\section{Conclusions}

In this paper, the TDFFD algorithm was extended to TMRI sequences. Preliminar motion and deformation results were reported for the set of 15 healthy volunteers and the phantom of the $1^{\text {st }} \mathrm{cMAC}$ database. On the phantom the weighting between non-sequential (prone to correct drift) and sequential (sensitive to accurate frame to frame tracking) was critical for the obtention of correct tracking results. We observed for healthy volunteers the uniformity of the strain pattern among different segments of the LV, in accordance with clinical literature. The current quantification results exclude the first frames. These could potentially be taken into account by segmenting the SSFP images for generating regions of interest when doing the tracking.

\section{References}

1. Fischer, S.E., McKinnon, G.C., Maier, S.E., Boesiger, P.: Improved myocardial tagging contrast. Magnetic Resonance in Medicine 30(2), 191-200 (1993)

2. Moore, C.C., Lugo-Olivieri, C.H., McVeigh, E.R., Zerhouni, E.A.: Threedimensional systolic strain patterns in the normal human left ventricle: Characterization with tagged MR imaging. Radiology 214(2), 453-466 (2000)

3. Rutz, A.K., Ryf, S., Plein, S., Boesiger, P., Kozerke, S.: Accelerated whole-heart 3D CSPAMM for myocardial motion quantification. Magnetic Resonance in Medicine 59(4), 755-763 (2008)

4. De Craene, M., Piella, G., Duchateau, N., Silva, E., Doltra, A., Gao, H., D'hooge, J., Camara, O., Brugada, J., Sitges, M., Frangi, A.F.: Temporal diffeomorphic freeform deformation for strain quantification in 3D-US images. In: MICCAI, LNCS 6362(2), pp. 1-8, Springer (2010)

5. Tobon-Gomez, C., De Craene, M., Dahl, A., Kapetanakis, S., Carr-White, G., Lutz, A., Rasche, V., Etyngier, P., Schaeffter, T., Riccobene, C., Martelli, Y., Camara, O., Frangi, A.F., Rhode, K.S: A multimodal database for the 1st cardiac motion analysis challenge. In: Proc. STACOM 2011, workshop held in Conjunction with MICCAI 2011. Submitted (2011)

6. Ordas, S., Oubel, E., Leta, R., Carreras, F., Frangi, A.F.: A statistical shape model of the whole heart and its application to model-based segmentation. In: SPIE Medical Imaging: Physiology, function, and structure from medical images 6511, pp. 65111K (2007)

7. Tutte, W.T. How to draw a graph. In: Proc. London Math. Soc. 13(3), pp. 743-768 (1963) 